Di sr upt $i$ on of vascul ar endot hel $i$ al honeost asi s i n systemi $\mathrm{j}$ uveni I e i di opathi $\mathrm{c}$ arthri t i s- associ at ed macr ophage act $i$ vat $i$ on syndr ome: The dynamic rol es of angi opoi et in- 1 and -2

\begin{tabular}{|l|l|}
\hline 著者 & 田? 優子 \\
\hline 著者別表示 & Tasaki Yuko \\
\hline $\begin{array}{l}\text { j our nal or } \\
\text { publ i cat i on t i t I e }\end{array}$ & 博士論文本文Ful I \\
\hline 学位授与番号 & 13301甲第4508号 \\
\hline 学位名 & 博士 (医学 $)$ \\
\hline 学位授与年月日 & 2017- 03- 22 \\
\hline URL & ht t p: //hdl . handl e. net /2297/48173 \\
\hline
\end{tabular}




\title{
Disruption of vascular endothelial homeostasis in systemic juvenile idiopathic arthritis-associated macrophage activation syndrome: The dynamic roles of angiopoietin-1 and -2
}

\author{
Yuko Tasaki $^{a}$, Masaki Shimizu ${ }^{\mathrm{a}, *}$, Natsumi Inoue $^{\mathrm{a}}$, Mao Mizuta $^{\mathrm{a}}$, Yasuo Nakagishi ${ }^{\mathrm{b}}$, Taizo Wada $^{\mathrm{a}}$, \\ Akihiro Yachie $^{\text {a }}$ \\ ${ }^{a}$ Department of Pediatrics, School of Medicine, Institute of Medical, Pharmaceutical, and Health Sciences, Kanazawa University, Japan \\ ${ }^{\mathrm{b}}$ Department of Pediatric Rheumatology, Hyogo Prefectural Kobe Children's Hospital, Japan
}

\section{A R T I C L E I N F O}

\section{Article history:}

Received 12 August 2015

Received in revised form 14 January 2016

Accepted 15 February 2016

Available online 22 February 2016

\section{Keywords:}

Systemic juvenile idiopathic arthritis

Macrophage activation syndrome

Angiopoietin 1

Angiopoietin 2

Endothelial function

\begin{abstract}
A B S T R A C T
To assess the role of angiopoietin (Ang)-1 and Ang-2 and to investigate the clinical significance of serum levels of them in systemic juvenile idiopathic arthritis (s-JIA)-associated macrophage activation syndrome (MAS), we determined these levels in 51 patients with s-JIA, 11 patients with polyarticular JIA (poly-JIA), 12 patients with virus associated hemophagocytic syndrome (VAHS), 12 patients with Kawasaki disease (KD), and 15 age-matched healthy controls (HC). The results were compared with clinical features of MAS. During the MAS phase, serum Ang- 1 levels were significantly decreased compared with those during the active and inactive phases. Serum Ang-2/1 ratio were significantly elevated during the MAS phase, compared with those during the active and inactive phases. There was a rapid increase in the Ang-2/1 ratio at the onset of MAS. Serum Ang- 1 and the Ang-2/1 ratio significantly correlated with measures of disease activity, including AST and LDH. Ang-2/1 dysregulation was also observed in patients with VAHS, whereas not observed in most cases of KD. The homeostasis of vascular endothelial function by Ang- 1 and Ang- 2 is disrupted in MAS. Serum Ang- 1 levels and the Ang-2/1 ratio might represent promising indicators of disease activity for MAS.
\end{abstract}

(c) 2016 Elsevier Ltd. All rights reserved.

\section{Introduction}

Systemic juvenile idiopathic arthritis (s-JIA) is a unique subtype of JIA, characterized by arthritis and other systemic features including spiking fever, salmon colored skin rash, hepatosplenomegaly, generalized lymphadenopathy, and polyserositis [1]. Recent studies have shown that s-JIA might be driven by innate proinflammatory cytokines. In particular, interleukin (IL)-1, IL-6, IL-18 play an important roles in the pathogenesis of s-JIA [2,3]. Furthermore, biological therapies that block these cytokines have dramatic effects in patients with s-JIA [4,5]. These findings support the hypothesis that s-JIA is an autoinflammatory condition.

Abbreviations: Ang, angiopoietin; s-JIA, systemic juvenile idiopathic arthritis; MAS, macrophage activation syndrome; poly-JIA, polyarticular juvenile idiopathic arthritis; VAHS, virus associated hemophagocytic syndrome; KD, Kawasaki disease; HC, healthy control; IL, interleukin.

* Corresponding author at: Department of Pediatrics, School of Medicine, Institute of Medical, Pharmaceutical, and Health Sciences, Kanazawa University, 13-1 Takaramachi, Kanazawa 920-8641, Japan.

E-mail address: shimizum@staff.kanazawa-u.ac.jp (M. Shimizu).
Macrophage activation syndrome (MAS) is a potentially fatal condition of S-JIA, characterized by fever, cytopenias, hepatosplenomegaly, lymphadenopathy, liver dysfunction, coagulopathy, and central nervous system dysfunction [6]. MAS is a secondary form of hemophagocytic lymphohistiocytosis (HLH) and massive hypercytokinemia induced by excess activation of macrophages and proliferation of T lymphocytes is closely associated with the development of MAS [6].

Vascular endothelial cells activated by inflammatory cytokines act as a procoagulant surface and contribute to intravascular coagulation of s-JIA [2]. Angiopoietins (Ang)-1 and -2 are key regulators of endothelial cell function [7,8]. In inflammatory conditions including sepsis and hemolytic uremic syndrome, the Ang-2/1 ratio increases because of decreased Ang-1, increased Ang-2 [9-12]. We speculated that dysregulation of the Ang-2/1 is also present in sJIA, and that the homeostasis of vascular endothelial function by Ang- 1 and Ang-2 is disrupted in MAS.

To assess the role of Ang- 1 and Ang- 2 in endothelial damage in the pathogenesis of s-JIA and MAS, we measured serum Ang- 1 and Ang-2 levels in patients with s-JIA. We compared them with the 
levels in patients with virus associated hemophagocytic syndrome (VAHS), a secondary form of HLH as well as MAS and Kawasaki disease (KD), a popular pediatric vasculitis which complicates the vascular endothelial damage. We determined the correlation between the levels of serum Ang-1and Ang-2 levels, and the Ang-2/1 ratio with measures of disease activity and severity in order to clarify the clinical significance of these levels as indicators of disease activity for MAS.

\section{Materials and methods}

\subsection{Patients and samples}

Serum samples were obtained from 51 patients with s-JIA, 11 patients with polyarticular JIA (poly-JIA), 12 patients with Kawasaki disease (KD), and 15 age-matched healthy controls (HC) (mean age s-JIA: $7.9 \pm 5.4$ years, mean age poly-JIA: $11.8 \pm 5.0$ years, mean age KD: $1.5 \pm 1.4$ years, mean age HC: $9.3 \pm 7.8$ years). Plasma samples were obtained from 12 patients with virus associated hemophagocytic syndrome (VAHS) (mean age VAHS: $5.0 \pm 4.0$ years). Eleven patients with s-JIA had MAS, and seven of these had already developed MAS by the time they were referred to us at the onset of s-JIA. Four patients with s-JIA developed MAS during the active phase after beginning immunosuppressive therapy with steroids and/or tocilizumab. Samples from these four patients were obtained during the active and inactive phases of sJIA. The clinical characteristics of the patients with active s-JIA are shown in Table 1 . The clinical characteristics of the patients with MAS are shown in Supplementary Table 1. Furthermore, samples from 16 patients with s-JIA were obtained during the active and inactive phases of s-JIA. The diagnoses of s-JIA and poly-JIA were based on the criteria of the International League of Associations for Rheumatology [13]. MAS was diagnosed based on the guidelines proposed by Ravelli et al [14]. The criteria for the active phase of s-JIA were defined as follows: active arthritis, fever, salmon colored rash, hepatosplenomegaly, generalized lymphadenopathy, polyserositis, increased erythrocyte sedimentation rate, and increased serum C-reactive protein (CRP) level. The criteria for the inactive phase of s-JIA on medication were as follows: the absence of clinical symptoms observed in the active phase of sJIA, normal erythrocyte sedimentation rate, and normal serum CRP level. The diagnosis of VAHS was made according to established HLH diagnostic criteria [15]. The diagnosis of KD was based on the classic clinical criteria [16].

\section{Table 1}

Clinical characteristics of patients with active systemic juvenile idiopathic arthritis. CRP, C-reactive protein; AST, aspartate aminotransferase; LDH, lactate dehydrogenase; FDP-DD, fibrin degradation product D-dimer; PSL, prednisolone; CyA, cyclosporine; MTX, methotrexate; TCZ, tocilizumab.

\begin{tabular}{ll}
\hline Patients & 44 \\
Sex & Male 20 female 24 \\
Age (years) & $8.4 \pm 5.5$ \\
Disease duration (months) & $11.2 \pm 39.0$ \\
Laboratory findings & \\
CRP $(\mathrm{mg} / \mathrm{dl})(n=43)$ & $10.1 \pm 7.1$ \\
AST $(\mathrm{IU} / \mathrm{L})(n=48)$ & $47.5 \pm 33.1$ \\
LDH $(\mathrm{IU} / \mathrm{L})(n=48)$ & $367.1 \pm 150.2$ \\
Ferritin $(\mathrm{ng} / \mathrm{ml})(n=48)$ & $1669.4 \pm 2926.7$ \\
FDP-DD $(n=34)$ & $6.1 \pm 7.0$ \\
Treatment & \\
PSL $(\mathrm{mg} / \mathrm{kg} /$ day) $(n=13)$ & $0.77 \pm 0.55$ \\
CyA $(\mathrm{mg} / \mathrm{kg} /$ day $)(n=5)$ & $2.7 \pm 1.6$ \\
MTX $\left(\mathrm{mg} / \mathrm{m}^{2} /\right.$ week $)(n=1)$ & 10 \\
TCZ $(\mathrm{mg} / \mathrm{kg} / 2$ weeks $)(n=5)$ & 8 \\
\hline
\end{tabular}

Sera and plasma were separated from the cells, divided into aliquots, frozen, and stored at $-80{ }^{\circ} \mathrm{C}$ until use. This study was approved by the Institutional Review Board at Kanazawa University, and all specimens were used only after informed consent was obtained according to the Declaration of Helsinki.

\subsection{Quantification of serum cytokines}

Levels of Ang-1 and Ang-2 were evaluated using enzyme-linked immunosorbent assay (ELISA), according to the manufacturer's instructions (RayBio Human Angiopoietin-1, -2 ELISA kit, RayBiotech, Norcross, GA, USA). The levels of Ang-1 and Ang-2 could be measured equally well in plasma and serum samples.

\subsection{Statistical analysis}

Within-group comparisons were analyzed using the MannWhitney test or paired $t$-test. Correlations were analyzed using the Spearman rank correlation coefficient. Analyzed measures with $p$ values $<0.05$ were considered to be statistically significant.

\section{Results}

3.1. Disruption of vascular endothelial homeostasis by Ang-1 and Ang2 in S-JIA and MAS

The serum Ang-1 and Ang-2 levels were determined in patients with s-JIA, and compared with the levels in patients with poly-JIA, VAHS, KD and in HC. As shown in Fig. 1A, during the MAS phase in patients with s-JIA, the serum Ang-1 levels were significantly decreased $(41,583 \pm 27,773 \mathrm{pg} / \mathrm{ml})$ compared with those during the active $(100,866 \pm 42,949 \mathrm{pg} / \mathrm{ml}, p=0.0001)$ and inactive phases $(120,553 \pm 33,076 \mathrm{pg} / \mathrm{ml}, p<0.0001)$ in patients with s-JIA, and compared with those in patients with poly-JIA $(101,850 \pm 25,951 \mathrm{pg} / \mathrm{ml}, p<0.01)$, in KD $(114,517 \pm 23,080 \mathrm{pg} / \mathrm{ml}$, $p<0.001)$ and in HC $(100,740 \pm 31,718 \mathrm{pg} / \mathrm{ml}, p<0.001)$. Serum Ang-1 levels in patients with VAHS were also significantly decreased $(41,583 \pm 27,773 \mathrm{pg} / \mathrm{ml})$ compared with those during the active and inactive phases in patients with s-JIA, and compared with those in patients with poly-JIA, in KD and in $\mathrm{HC}$ $(100,740 \pm 31,718 \mathrm{pg} / \mathrm{ml}, p<0.001)$. As shown in Fig. 1B, during the MAS phase in patients with s-JIA, serum Ang-2 levels were significantly elevated $(8750 \pm 7488 \mathrm{pg} / \mathrm{ml})$ compared with those during the active $(5514 \pm 5392 \mathrm{pg} / \mathrm{ml}, p<0.05)$ and inactive phases $(2836 \pm 1341 \mathrm{pg} / \mathrm{ml}, p<0.01)$ in patients with s-JIA, and compared with those in patients with poly-JIA $(2631 \pm 1554 \mathrm{pg} / \mathrm{ml}, p<0.05)$, in VAHS $(5225 \pm 6784 \mathrm{pg} / \mathrm{ml}, p<0.05)$, in $\mathrm{KD}(4191 \pm 3704 \mathrm{pg} / \mathrm{ml}$, $p<0.01)$ and in HC $(2317 \pm 1035 \mathrm{pg} / \mathrm{ml}, p<0.001)$. As shown in Fig. $1 \mathrm{C}$, during the MAS phase in patients with s-JIA, the Ang-2/1 ratio was significantly elevated $(0.272 \pm 0.254 \mathrm{pg} / \mathrm{ml})$ compared with that during the active $(0.066 \pm 0.071 \mathrm{pg} / \mathrm{ml}, p<0.001)$ and inactive phases $(0.026 \pm 0.012 \mathrm{pg} / \mathrm{ml}, p<0.0001)$ in patients with s-JIA, and compared with those in patients with poly-JIA $(0.028 \pm 0.019 \mathrm{pg} / \mathrm{ml}, \quad p<0.001), \quad$ in $\mathrm{KD}(0.036 \pm 0.028 \mathrm{pg} / \mathrm{ml}$, $p<0.0001)$ and in HC $(0.023 \pm 0.007 \mathrm{pg} / \mathrm{ml}, p<0.0001)$. Furthermore, as shown in Fig. $1 \mathrm{C}$, during the active phase, the Ang-2/1 ratio was significantly elevated compared with that during the inactive phase in patients with s-JIA $(p<0.01)$, and compared with that in patients with poly-JIA $(p<0.05)$ and in HC $(p<0.01)$ (Fig. 1C). However, the Ang-2/1 ratio during the active phase of s-JIA were significantly decreased compared with those in patients with VAHS $(0.165 \pm 0.196 \mathrm{pg} / \mathrm{ml}, p<0.05)$. 


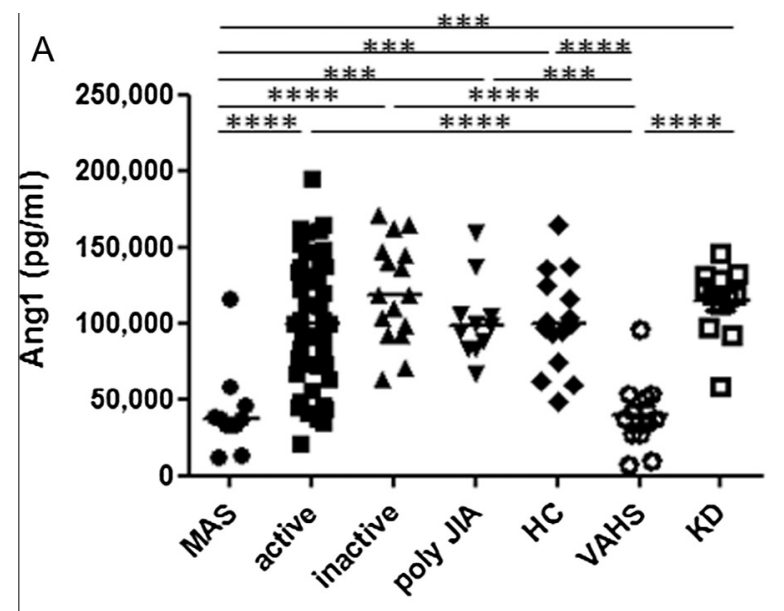

3.2. The time course of changes in serum Ang-1 levels and the Ang-2/1 ratio in four patients with MAS

To investigate the relevance of Ang- 1 and Ang-2 in the pathogenesis of MAS, serum Ang-1 and Ang-2 levels were serially monitored in four cases of MAS (Fig. 2A-D). The serum Ang-1 levels profoundly and rapidly decreased as MAS developed. However, the Ang-2/1 ratio profoundly and rapidly increased as MAS developed. The Ang-2/1 ratio subsequently decreased after such manifestations disappeared with immunosuppressive therapy. As shown in Fig. 2A and D, in cases of uncontrolled disease activity, serum Ang-1 levels were decreased and the Ang-2/1 ratio was elevated.

3.3. Correlation between serum Ang-1 and Ang-2 levels and Ang-2/1 ratio and measures of disease activity in patients with s-JIA and MAS

Because serum CRP, aspartate aminotransferase (AST), lactate dehydrogenase (LDH), and ferritin levels are clinically used as indicators of disease activity in s-JIA, these levels were compared with the serum Ang-1 and Ang-2 levels and the Ang-2/1 ratio. Serum Ang-1 levels negatively correlated with AST, LDH, and ferritin (Fig. 3A-C). Serum Ang-2 levels positively correlated with AST and $\mathrm{LDH}$ (Fig. 3D and E). The Ang-2/1 ratio positively correlated with AST, LDH, and ferritin (Fig. 4A-C). Furthermore, levels of the plasma fibrin degradation product D-dimer (FDP-DD) are clinically used as indicators of coagulopathy. Therefore, FDP-DD concentrations were compared with serum Ang-1 and Ang-2 levels and the Ang-2/1 ratio. The Ang-2/1 ratio positively correlated with FDPDD concentrations (Fig. 4D).

\section{Discussion}

Ang-1 and Ang-2 competitively bind to the endothelial Tie-2 receptor [7,8]. Ang-1 is constitutively produced by pericytes, smooth muscle cells and fibroblast. Ang-2 is produced by endothelial cells. In healthy individuals, Ang-2 expression is almost absent in the resting vasculature. Serum concentrations of Ang- 1 exceed those of Ang-2 and Ang2/1 ratio is low. Ang- 1 binds to the Tie-2 receptor and control vascular quiescence through the negative regulation of proinflammatory pathways $[7,8]$. In inflammatory conditions, Ang-2 expression is dramatically induced by various stresses. Ang-2 is released from endothelial cells and competes with Ang-1 for binding to the Tie-2 receptor. Consequently, Ang-2 triggers proinflammatory and prothrombotic pathways by activating endothelial cells.

Various stresses including hypoxia, inflammation, and high glucose concentrations induce Ang2/1 dysregulation [7,8]. Cytokines and growth factors including tumor necrosis factor (TNF)- $\alpha$, vascular endothelial growth factor, and fibroblast growth factor promote destabilized angiogenesis by modulating angiopoietin expression $[7,8]$. Among the cytokines closely related to the pathogenesis of s-JIA, previous studies have shown that IL-1 $\beta$ downregulates Ang-1 expression [17]. Furthermore, IL-6 inhibits Ang-1 signaling by downregulating Ang-1 expression and upregulating Ang-2 expression $[18,19]$.

In this study, we demonstrated that the Ang-2/1 ratio, which Fig. 1. Serum angiopoietin (Ang)- 1 (A) and -2 (B) levels and the Ang-2/1 ratio (C) in patients with systemic juvenile idiopathic arthritis. Bars represent median values. Ang-1, angiopoietin-1; Ang-2, angiopoietin-2; poly-JIA, polyarticular juvenile idiopathic arthritis; VAHS, virus associated hemophagocytic syndrome; KD, Kawasaki disease; HC, healthy controls. Statistically significant differences between each patient group are shown as, ${ }^{*} P<0.05,{ }^{* *} P<0.01,{ }^{* * *} P<0.001$, and ${ }^{* * * *} P<0.001$. reflects a relative excess of Ang-2, was significantly elevated in the active phase of s-JIA. Furthermore, serum Ang- 1 levels were significantly decreased in MAS, whereas the serum Ang-2 levels and the Ang-2/1 ratio were significantly elevated in MAS. These findings are consistent with the known biological roles of Ang-1 

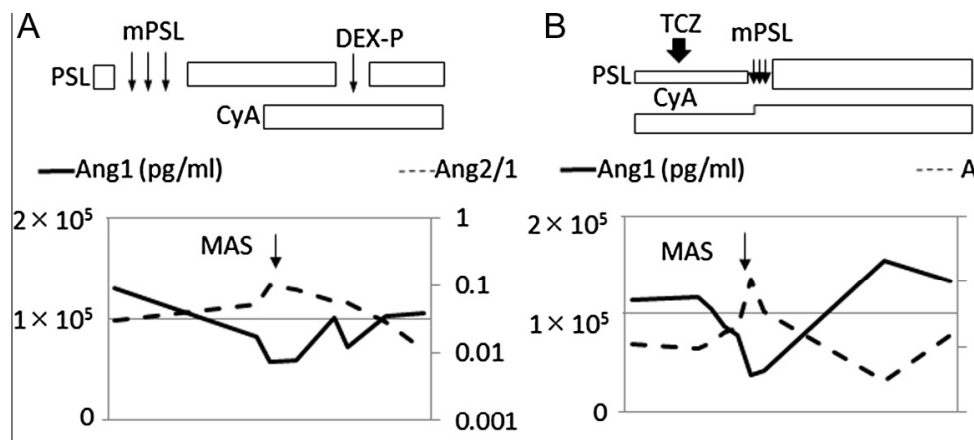

一Ang1 (pg/ml)

-..- Ang2/1
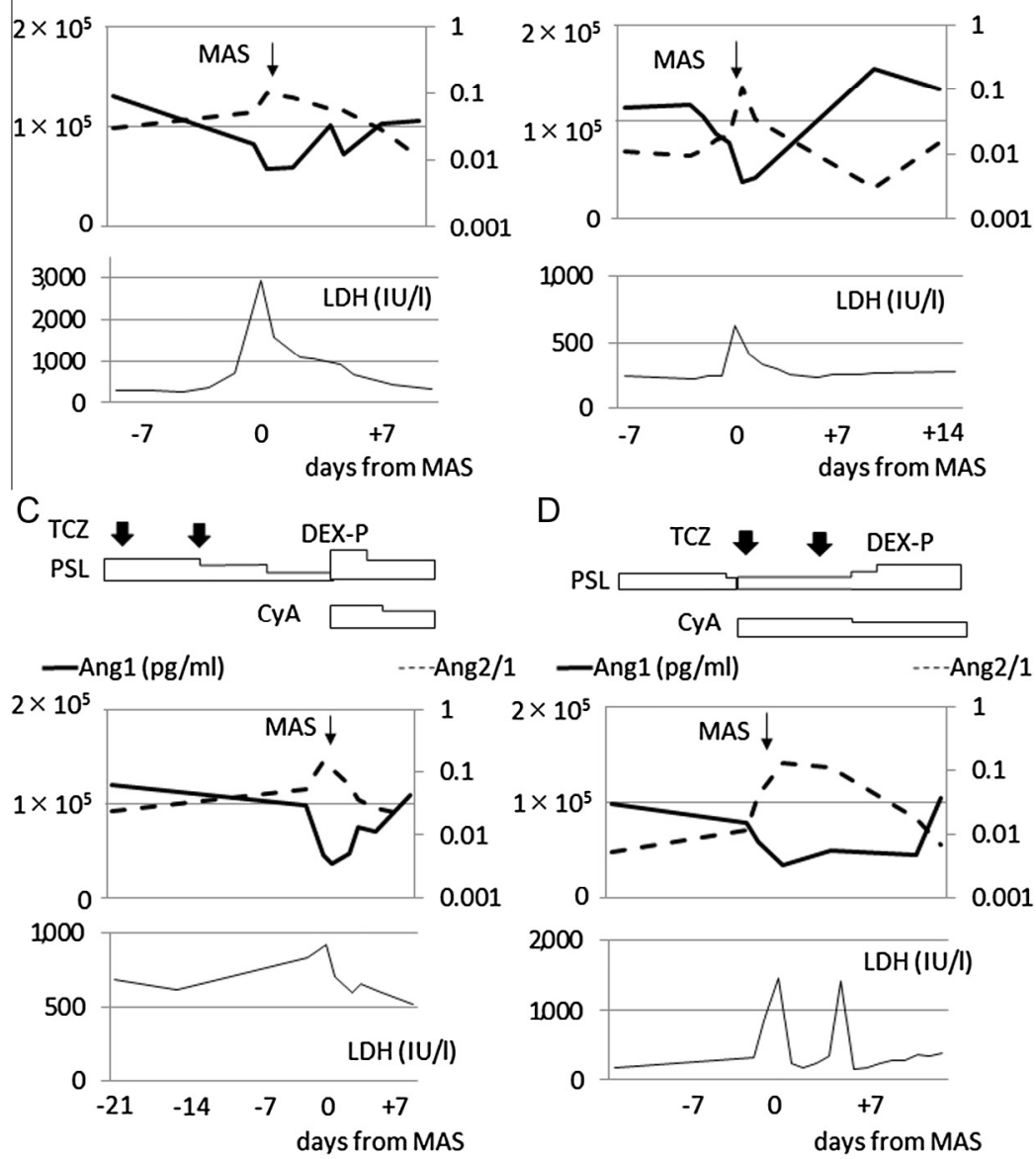

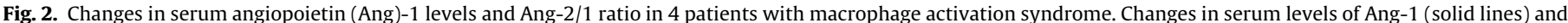

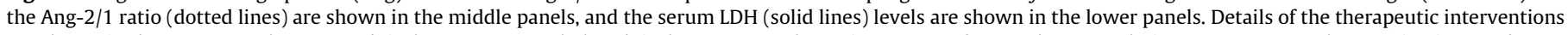

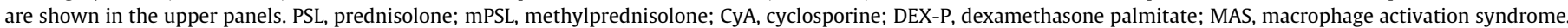

and Ang-2, and their predicted impact on endothelial cell function in the pathogenesis of s-JIA and MAS. In agreement with these findings, endothelial cell dysfunction by Ang-2/1 dysregulation is present in children with s-JIA and represents the clinical manifestation of endothelial cell dysfunction. In the pathology of s-JIA, proinflammatory cytokines, such as IL-1 $\beta$ and IL-6, induce Ang2/1 dysregulation through downregulation of Ang-1 expression and upregulation of Ang-2 expression. Furthermore, the homeostasis of endothelial function by Ang-1 and Ang-2 is disrupted as MAS develops, inducing vascular endothelial cell damage and coagulopathy. Interestingly, Ang-2/1 dysregulation was also observed in patients with VAHS, a secondary form of HLH as well as MAS. In contrast, Ang-2/1 dysregulation was not observed in most cases of KD. From these findings, Ang-2/1 dysregulation is characteristic to the diseases with progressive endothelial dysfunction such as MAS and HLH.

In this study, the Ang-2/1 ratio profoundly and rapidly increased as MAS developed. Furthermore, the Ang-2/1 ratio decreased after such manifestations disappeared with corticosteroid and cyclosporine therapy. As shown in Fig. 1A and D, serum Ang- 1 levels were decreased and the Ang-2/1 ratio was elevated in cases of uncontrolled disease activity. From these findings, the Ang-2/1 ratio may represent a promising indicator of disease activity for MAS.
In addition to the role of Ang-2/1 in the homeostasis of endothelial function, several lines of evidence have suggested that Ang-1 and Ang-2 play roles in the exacerbation of synovitis in patients with rheumatoid arthritis (RA) [18,20,21]. Ang-1, Ang-2, and Tie2, are expressed in RA synovial tissue [20,21]. In the synovial tissue of RA patients, Tie2 activation has been shown to be predominantly localized to macrophages [18]. Ang- $1 / 2$ has been shown to stimulate the activation of intracellular signaling pathways, and cooperate with TNF to induce macrophage IL-6 and macrophage inflammatory protein $1 \alpha$ production [21]. These findings indicate that the synovial macrophage is a primary target of Ang-signaling, promoting the proinflammatory activation of human macrophages. In animal models of RA, neutralization of Ang-2 was shown to significantly decrease disease severity, synovial inflammation, neo-vascularization, and joint destruction [21]. From these findings, Ang-1 and Ang-2 may play important roles in the development of arthritis in S-JIA and RA. Furthermore, direct targeting of Ang-2 may provide therapeutic benefit in the treatment of s-JIA-associated MAS.

The limitation of the present study was the small number of patients with s-JIA. Further larger studies may help to define the true diagnostic value of Ang -1 and Ang-2. However, despite this limitation, our results suggest that Ang-1 and Ang-2 play important roles in the complex network involved in the inflammation 

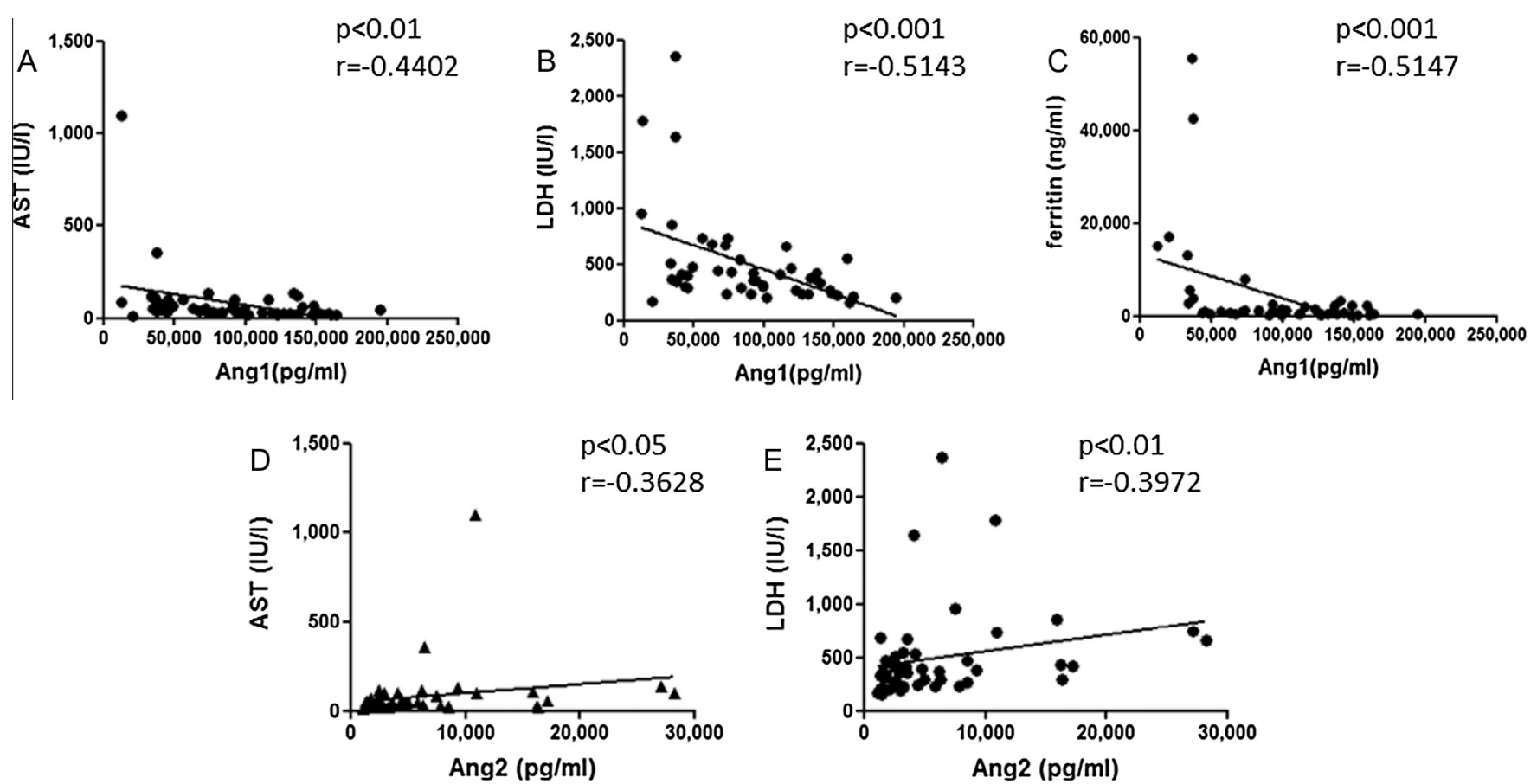

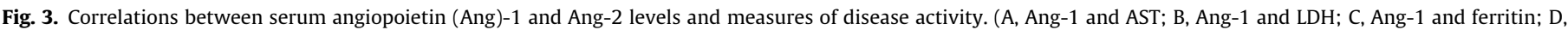
Ang-2 and AST; E, Ang-2 and LDH). AST, aspartate aminotransferase; LDH, lactate dehydrogenase.
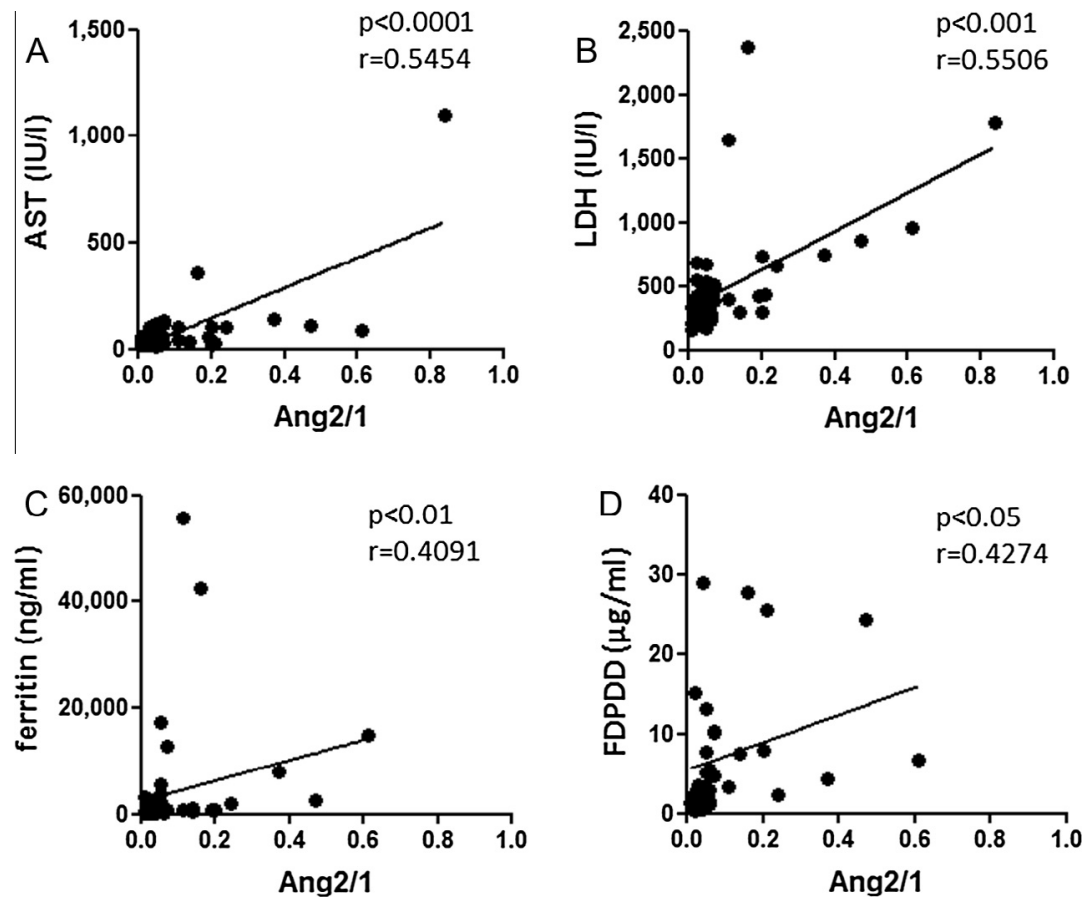

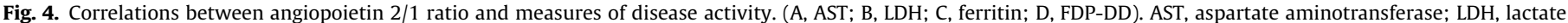
dehydrogenase; FDP-DD, fibrin degradation product D-dimer.

of s-JIA and MAS. The homeostasis of endothelial function by Ang-1 and Ang-2 is disrupted in MAS. The Ang-2/1 ratio may represent a promising indicator of disease activity for MAS.

\section{Funding source}

No external funding was granted to this study.

\section{Financial disclosure}

The authors have no financial relationship to this article to disclose.

\section{Conflict of interest}

The authors have no conflicts of interest to disclose. 


\section{Acknowledgment}

We thank Harumi Matsukawa for technical assistance.

\section{Appendix A. Supplementary material}

Supplementary data associated with this article can be found, in the online version, at http://dx.doi.org/10.1016/j.cyto.2016.02.008.

\section{References}

[1] G.F. Still, On a form of chronic joint disease in children, Med. Chir. Trans. 80 (1897) 47-59.

[2] E.D. Mellins, C. Macaubas, A.A. Grom, Pathogenesis of systemic juvenile idiopathic arthritis: some answers, more questions, Nat. Rev. Rheumatol. 7 (2011) 416-426.

[3] M. Shimizu, T. Yokoyama, K. Yamada, et al., Distinct cytokine profiles of systemic-onset juvenile idiopathic arthritis-associated macrophage activation syndrome with particular emphasis on the role of interleukin-18 in its pathogenesis, Rheumatology 49 (2010) 1645-1653.

[4] S. Yokota, T. Imagawa, M. Mori, et al., Efficacy and safety of tocilizumab in patients with systemic-onset juvenile idiopathic arthritis: a randomised, double-blind, placebo-controlled, withdrawal phase III trial, Lancet 371 (2008) 998-1006.

[5] N. Ruperto, H.I. Brunner, P. Quartier, et al., Two randomized trials of canakinumab in systemic juvenile idiopathic arthritis, N. Engl. J. Med. 367 (2012) 2396-2406

[6] A. Ravelli, A.A. Grom, E.M. Behrens, R.Q. Cron, Macrophage activation syndrome as part of systemic juvenile idiopathic arthritis: diagnosis, genetics, pathophysiology and treatment, Genes Immun. 13 (2012) 289-298.

[7] U. Fiedler, H.G. Augustin, Angiopoietins: a link between angiogenesis and inflammation, Trends Immunol. 27 (2006) 552-558.

[8] L. Eklund, P. Saharinen, Angiopoietin signaling in the vasculature, Exp. Cell Res. 319 (2013) 1271-1280.

[9] P. Kumpers, A. Lukasz, S. David, et al., Excess circulating angiopoietin-2 is a strong predictor of mortality in critically ill medical patients, Crit. Care 12 (2008) R147.
[10] A.V. Page, M. Kotb, A. McGeer, et al., Systemic dysregulation of angiopoietin-1/ 2 in streptococcal toxic shock syndrome, Clin. Infect. Dis. 52 (2011) e157e161.

[11] F.E. Lovegrove, N. Tangpukdee, R.O. Opoka, et al., Serum angiopoietin-1 and -2 levels discriminate cerebral malaria from uncomplicated malaria and predict clinical outcome in African children, PLoS ONE 4 (2009) e4912.

[12] A.V. Page, P.I. Tarr, S.L. Watkins, et al., Dysregulation of angiopoietin 1 and 2 in Escherichia coli 0157:H7 infection and the hemolytic-uremic syndrome, J. Infect. Dis. 208 (2013) 929-933.

[13] R.E. Petty, T.R. Southwood, P. Manners, et al., International League of Associations for Rheumatology classification of juvenile idiopathic arthritis: second revision, Edmonton, 2001, J. Rheumatol. 31 (2004) 390-392.

[14] A. Ravelli, S. Magni-Manzoni, A. Pistorio, et al., Preliminary diagnostic guidelines for macrophage activation syndrome complicating systemic juvenile idiopathic arthritis, J. Pediatr. 146 (2005) 598-604.

[15] A.H. Filipovich, Hemophagocytic lymphohistiocytosis (HLH) and related disorders, Hematol. Am. Soc. Hematol. Educ. Program (2009) 127-131.

[16] J.W. Newburger, M. Takahashi, M.A. Gerber, et al., Diagnosis, treatment, and long-term management of Kawasaki disease: a statement for health professionals from the Committee on Rheumatic Fever, Endocarditis and Kawasaki Disease, Council on Cardiovascular Disease in the Young, American Heart Association, Pediatrics 114 (2004) 1708-1733.

[17] F. Fan, O. Stoeltzing, W. Liu, et al., Interleukin-1beta regulates angiopoietin-1 expression in human endothelial cells, Cancer Res. 64 (2004) 3186-3190.

[18] K. Kayakabe, T. Kuroiwa, N. Sakurai, et al., Interleukin-6 promotes destabilized angiogenesis by modulating angiopoietin expression in rheumatoid arthritis, Rheumatology 51 (2012) 1571-1579.

[19] I. Kim, J.H. Kim, Y.S. Ryu, M. Liu, G.Y. Koh, Tumor necrosis factor-alpha upregulates angiopoietin-2 in human umbilical vein endothelial cells, Biochem. Biophys. Res. Commun. 269 (2000) 361-365.

[20] K. Takahara, T. Iioka, K. Furukawa, et al., Autocrine/paracrine role of the angiopoietin-1 and -2/Tie2 system in cell proliferation and chemotaxis of cultured fibroblastic synoviocytes in rheumatoid arthritis, Human Pathol. 35 (2004) 150-158.

[21] S. Krausz, S. Garcia, C.A. Ambarus, et al., Angiopoietin-2 promotes inflammatory activation of human macrophages and is essential for murine experimental arthritis, Ann. Rheum. Dis. 71 (2012) 1402-1410. 